\section{Raman Spectrum of Deuterobenzene}

WE have photographed the Raman spectra of the various deuterobenzenes. In order to obtain the isotopic shifts of the Raman frequencies with a fair accuracy, the spectra were investigated with high dispersion, which necessitates a rather large volume of the substance; the Raman tube employed contained 18 c.c. For the preparation of deuterobenzene we have investigated various possible exchange reactions and have finally chosen the Friedel-Craft reaction :

$$
\mathrm{C}_{6} \mathrm{H}_{6}+6 \mathrm{DCl} \underset{\mathrm{AlCl}_{3}}{\rightleftarrows} \mathrm{C}_{6} \mathrm{D}_{6}+6 \mathrm{HCl}
$$

This process has the advantage of high reaction velocity, and further, our experiments indicate an appreciable shift of the equilibrium towards the deuterobenzene. Details concerning this reaction as well as the common physical properties of the deuterobenzene will appear elsewhere. Of course this method produces a mixture of the various deuterosubstituted benzenes in proportions very nearly corresponding to the probability distribution. By photographing the spectra of deuterobenzenes with various contents of deuterium, it is consequently possible to identify the Raman lines belonging to each deuterobenzene.

We have measured the com. plete Raman spectra of the molecules $\mathrm{C}_{6} \mathrm{H}_{5} \mathrm{D}, \mathrm{C}_{6} \mathrm{D}_{5} \mathrm{H}$ and $\mathrm{C}_{6} \mathrm{D}_{6}$. All the Raman frequencies of benzene are found to be lowered by introduction of deuterium in the molecule.

Fig. 1 is an enlargement of a part of two Raman plates and shows the isotopic shift of the strongest line of the spectrum, namely, the frequency corresponding to the symmetrical ring vibration. The plates show distinctly greater isotopic shift between $\mathrm{C}_{6} \mathrm{H}_{6}$ and $\mathrm{C}_{6} \mathrm{H}_{5} \mathrm{D}$ than between $\mathrm{C}_{6} \mathrm{D}_{5} \mathrm{H}$ and $\mathrm{C}_{6} \mathrm{D}_{6}$, owing to the comparatively greater alteration in mass in the first

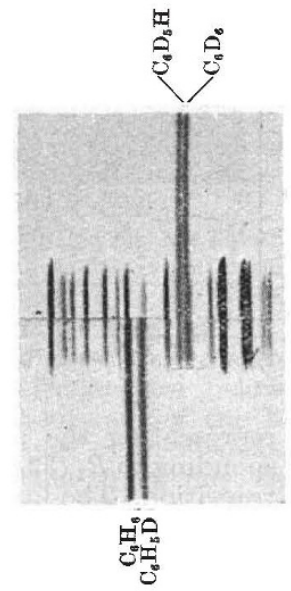

FIG. 1.
$\mathrm{C}_{6} \mathrm{H}_{6}$ and $\mathrm{C}_{6} \mathrm{D}_{8}$, and are of great interest for the identification of these. A detailed report of the investigation and a discussion of the results will appear shortly elsewhere.

\section{A. KLIT.}

A. Langseth.

Universitetets kemiske Laboratorium, København.

May 3.

\section{Spontaneous Emission of Neutrons by Radio-elements}

I. CURIE, Joliot and Preiswerk ${ }^{1}$ have shown that by bombarding silicon and phosphorus by neutrons there are produced radio-elements which spontaneously emit neutrons, in addition to electrons (Fermi effect), positrons and $\gamma$-rays. Thus an entirely new type of radioactivity has been discovered by them.

The mechanism of the spontaneous ejection of neutrons is not fully understood. At any rate, it may be supposed that this process is a secondary one, as the experiments on the Fermi effect have shown clearly enough that the potential energy of the neutron in the nuclear field is negative (the barrier is absent), and hence the neutron, having received somehow or other some positive energy, must leave the nucleus immediately or pass into a state of negative energy, exciting another particle or emitting $\gamma$-quanta.

In their communication, I. Curie, Joliot and Preiswerk explain the emission of the neutrons by the following processes :

(1) ${ }_{13} \mathrm{Al}^{28} \rightarrow{ }_{13} \mathrm{Al}^{27}+{ }_{0} n^{1}$ and $(2){ }_{14} \mathrm{Si}^{31} \rightarrow{ }_{14} \mathrm{Si}^{30}+{ }_{0} n^{1}$.

Such an interpretation (primary process) cannot be admitted according to the considerations mentioned above; moreover, it would seem impossible energetically, although we cannot assert this definitely because the accurate values of the masses of all the components of reactions 1 and 2 are not known.

I have attempted to find the energy distribution of the spontaneously ejected neutrons or at least to estimate its upper limit. For the detection of the neutrons I used a photographic plate with a thick layer of emulsion $(50 \mu)$ specially prepared for the registration of $\mathrm{H}$-particles. At a distance of $0 \cdot 2-0 \cdot 4$ $\mathrm{mm}$. of the plate, there was placed a paraffin film are respectively : $992 \cdot 6,981 \cdot 5,952 \cdot 4$ and $946 \cdot 6$ $\mathrm{cm} .^{-1}$.

The Raman frequencies of $\mathrm{C}_{6} \mathrm{D}_{6}$, together with those of $\mathrm{C}_{6} \mathrm{H}_{6}$, are as follows:

$$
\text { Raman Frequencies }\left(\mathrm{cm}^{-1}\right. \text {.) }
$$

$\mathrm{C}_{6} \mathrm{H}_{6} 606 \cdot 4,849 \cdot 7,992 \cdot 6,1175 \cdot 6,1585 \cdot 9$, $1604 \cdot 2,3048 \cdot 3,3061 \cdot 5$.

$\mathrm{C}_{6} \mathrm{D}_{6} 581 \cdot 6,844 \cdot 7,946 \cdot 6,869 \cdot 8,1555 \cdot 4$, $1569 \cdot 0,2266 \cdot 8,2292 \cdot 0$.

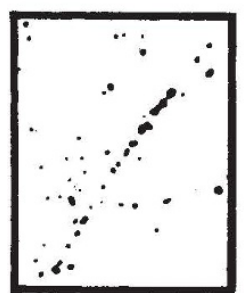

FIG. 1. Stereoscopic photomicrographs of the track of a recoil proton in photographic emulsion. Length of the track is $72 \mu\left(\sim 2.6 \times 10^{6} \mathrm{e} . \mathrm{v}\right.$. $)$
It is interesting to note the great shift of the 1175.6 line in benzene (to $869.8 \mathrm{~cm}^{-1}$ in $\mathrm{C}_{6} \mathrm{D}_{6}$ ), which shows that this frequency, as well as the 3048.3 and the 3061.5 frequencies, belongs to a pronounced hydrogen vibration.

The spectra of $\mathrm{C}_{6} \mathrm{H}_{5} \mathrm{D}$ and $\mathrm{C}_{6} \mathrm{D}_{5} \mathrm{H}$ are much more complicated owing to the split up of the degeneracy of certain vibrations in the symmetrical molecules and thus were registered the protons ejected by the neutrons from paraffin (Fig. 1). Radioactive isotopes were being produced in red phosphorus by neutron bombardment. A glass tube containing radon (about $250 \mathrm{mc}$.) and some powdered beryllium served as the source of neutrons. Every 10 minutes the bombarded phosphorus was brought into the immediate neighbourhood of the photographic plate for a period of 